\title{
Grand Challenges and Opportunities in Biophotonics
}

\author{
Bahman Anvari * \\ Department of Bioengineering, University of California, Riverside, Riverside, CA, United States
}

Keywords: biology, environment, light, lasers, microscopy, medicine, optical imaging, therapeutics

Biophotonics is the scientific field at the interface of life and light sciences. It encompasses the use of light as the energy source that enables fundamental studies and application developments in biological, pharmaceutical, environmental and agricultural sciences, and medicine. While the term is contemporary, some of the earliest recorded history of the field probably dates back to the 17th century with the first microbial observations by Antony van Leeuwenhoek using single lenses (Ford, 1985). In 1903, The Noble Prize in Physiology or Medicine was awarded to Niels Ryber Finsen "in recognition of his contribution to the treatment of diseases, especially lupus vulgaris, with concentrated light radiation, whereby he has opened a new avenue for medical science" ${ }^{\text {. Fast }}$ forward to 21st century where The Noble Prize in Chemistry was awarded "for the discovery and development of the green fluorescent protein" in 2008, and "for the development of super-resolved fluorescence microscopy"3 in 2014. The Noble Prize in Physics in 2018 was partly awarded to Arthur Ashkin "for the optical tweezers and their application to biological systems"4.

At the most fundamental level, the nature of the interaction of light with biological and organic matters provides the basis for both basic and translational work in biophotonics. These interactions involve the absorption and scattering of photons, and have led to the development of diverse technologies including various types of optical spectroscopy methods such as UV-VIS-IR spectroscopy, Fourier Transform Infrared Spectroscopy (FTIR), and Raman scattering; microscopy methods including confocal and multiphoton, fluorescence lifetime imaging (FLIM), and nanoscale optical microscopy (Hermann and Gordon, 2018); and clinical diagnostic and imaging technologies such as pulse oximetry, optical coherence tomography (OCT) (Huang et al., 1991), and fluorescence-guided surgery (Landau et al., 2016).

On the therapeutic side, Leon Goldman pioneered the use of lasers in dermatology (Goldman et al., 1963). Soon after, clinical applications of lasers were extended to ophthalmology for treatment of diabetic retinopathy (L'Esperance, 1969) and photodynamic therapy, and more recently to photoimmunotherapy (Kobayashi and Choyke, 2019; Xu et al., 2020). Optical neuromodulation including optogenetics and non-genetic photostimulation methods for optical manipulation of cellular and sub-cellular activities have recently emerged (Boyden et al., 2005; Jiang et al., 2019).

Despite these remarkable achievements and tremendous contributions to life sciences and medicine, there are significant challenges and yet exciting opportunities in biophotonics. Inherently, depth of optical penetration in biological materials remains limited to a few $\mathrm{cm}$. Furthermore, increased optical penetration depth is accompanied by decreased spatial resolution. Methodologies that can enable increased penetration depth and spatial resolution would have great impact in photo-therapeutics and optical imaging applications. In vivo measurements of optical

Received: 01 June 2021 Accepted: 11 June 2021 Published: 22 June 2021

Citation: Anvari B (2021) Grand Challenges and Opportunities in Biophotonics.

${ }^{1}$ The Nobel Prize in Physiology or Medicine 1903 [Online]. Available: https://www.nobelprize.org/prizes/medicine/1903/ summary/.

${ }^{2}$ The Nobel Prize in Chemistry 2008 [Online]. Available: https://www.nobelprize.org/prizes/chemistry/2008/summary/.

${ }^{3}$ The Nobel Prize in Chemistry 2014 [Online]. Available: https:/www.nobelprize.org/prizes/chemistry/2014/summary/.

${ }^{4}$ The Nobel Prize in Physics 2018 [Online]. Available: https://www.nobelprize.org/prizes/physics/2018/summary/. 
properties can lead to development of real time diagnostics, guided-therapies and evaluation of therapeutic interventions.

Multiplexed molecular sensing and development of new probes that can provide high sensitivity and specificity present another arena for further developments. Compact and miniatured devices, and wearable and implantable sensors would be of immense value for use at home, and at point-ofcare and resource-limited settings. The current COVID-19 pandemic illustrates the need for such practical, inexpensive, and easy-to-use devices that can provide rapid and accurate diagnostics.

Light-based theranostic technologies integrated with molecular and genomic profiling would provide capabilities for combined sensing/diagnostics/imaging and therapeutics on personalized basis. Photonic technologies will have important roles in high throughput drug screening, in vivo tracking of drugs biodistribution, and mediating localized and controlled-release of drugs. A better understanding of the immune response and the role of various inflammatory cells and signaling biomolecules to light can lead to development of more effective phototherapeutic methods.

The progress in artificial intelligence, including machine learning, data mining, big data analysis, and computational power provide opportunities for closer interactions and integrations with biophotonics toward automated feature and pattern identifications that may otherwise not be possible. Such interactions will also be increasingly useful for applications in

\section{REFERENCES}

Boyden, E. S., Zhang, F., Bamberg, E., Nagel, G., and Deisseroth, K. (2005). Millisecond-timescale, Genetically Targeted Optical Control of Neural Activity. Nat. Neurosci. 8, 1263-1268. doi:10.1038/nn1525

Ford, B. J. (1985). Single Lens: The story of the Simple Microscope. London: HarperCollins.

Goldman, L., Blaney, D. J., Kindel, D. J., Richfield, D., and Franke, E. K. (1963). Pathology of the Effect of the Laser Beam on the Skin. Nature 197, 912-914. doi:10.1038/197912b0

Hermann, R. J., and Gordon, M. J. (2018). Nanoscale Optical Microscopy and Spectroscopy Using Near-Field Probes. Annu. Rev. Chem. Biomol. Eng. 9, 356-387. doi:10.1146/annurev-chembioeng-060817-084150

Huang, D., Swanson, E., Lin, C., Schuman, J., Stinson, W., Chang, W., et al. (1991). Optical Coherence Tomography. Science 254, 1178-1181. doi:10.1126/ science.1957169

Jiang, Y., Parameswaran, R., Li, X., Carvalho-De-Souza, J. L., Gao, X., Meng, L., et al. (2019). Nongenetic Optical Neuromodulation with Silicon-Based Materials. Nat. Protoc. 14, 1339-1376. doi:10.1038/s41596-019-0135-9 environmental monitoring including the assessment of climate change and marine life, and in food and agricultural monitoring for pathogens and toxins detections as well as soil and vegetation evaluation. These examples highlight the multidisciplinary nature of biophotonics and the opportunities for collaborations among scientists with various expertise, as well as the need for development of educational curricula that emphasize multiple disciplines to train the future generations of scientists working in this field.

The immense capabilities of biophotonics have been recognized by specific government agencies to support biophotonics research. A particular example is the Biophotonics Program at United States National Science Foundation whose goal is "to explore the research frontiers in photonics principles, engineering and technology that are relevant for critical problems in fields of medicine, biology and biotechnology." Funding through appropriate governmental agencies will be needed to tackle these grand challenges and ultimately impact human health and the environment on our planet. The field of biophotonics continues to grow and embraces new interested scientists.

\section{AUTHOR CONTRIBUTIONS}

The author confirms being the sole contributor of this work and has approved it for publication.

Kobayashi, H., and Choyke, P. L. (2019). Near-Infrared Photoimmunotherapy of Cancer. Acc. Chem. Res. 52, 2332-2339. doi:10.1021/acs.accounts.9b00273

Landau, M. J., Gould, D. J., and Patel, K. M. (2016). Advances in Fluorescent-Image Guided Surgery. Ann. Transl. Med. 4, 392. doi:10.21037/atm.2016.10.70

L'esperance, F. A., Jr. (1969). Treatment of Ophtalmic Vascular Diseases by Argon Laser Photocoagulation. Trans. Am. Acad. Ophthalmol. Otolaryngol. 73, 1077-1096.

$\mathrm{Xu}, \mathrm{X}$., Lu, H., and Lee, R. (2020). Near Infrared Light Triggered Photo/immunoTherapy toward Cancers. Front. Bioeng. Biotechnol. 8, 488. doi:10.3389/ fbioe. 2020.00488

Conflict of Interest: The author declares that the research was conducted in the absence of any commercial or financial relationships that could be construed as a potential conflict of interest.

Copyright (c) 2021 Anvari. This is an open-access article distributed under the terms of the Creative Commons Attribution License (CC BY). The use, distribution or reproduction in other forums is permitted, provided the original author(s) and the copyright owner(s) are credited and that the original publication in this journal is cited, in accordance with accepted academic practice. No use, distribution or reproduction is permitted which does not comply with these terms. 